\title{
ALTERAÇÕES CARDIOVASCULARES E MORTE SÚBITA NAS EPILEPSIAS
}

\author{
Fulvio Alexandre Scorza', Marly de Albuquerque ${ }^{2}$, \\ Ricardo Mario Arida ${ }^{3}$, Esper Abrão Cavalheiro ${ }^{4}$
}

\begin{abstract}
RESUMO - A epilepsia é a doença neurológica crônica grave mais comum e o fenômeno da morte súbita nas epilepsias (SUDEP) é a causa direta de morte mais importante nesta doença. A causa da SUDEP ainda é desconhecida, no entanto, alterações cardiovasculares têm sido sugeridas como os mecanismos mais comuns. Sendo assim, enfatizamos nesta revisão a relação existente entre SUDEP e alterações cardiovasculares.
\end{abstract}

PALAVRAS-CHAVE: epilepsia, SUDEP, alterações cardiovasculares, drogas antiepilépticas.

\section{Cardiovascular alterations and sudden death in epilepsy}

ABSTRACT - Epilepsy is the most common neurological disorder and sudden unexpected death in epilepsy (SUDEP) is the most important direct epilepsy-related cause of death. The cause of SUDEP is still unknown, however, the most commonly suggested mechanisms are cardiac abnormalities. Based on this, in this review the relationship between SUDEP and cardiac abnormalities has been emphasized.

KEY WORDS: epilepsy, SUDEP, cardiac alterations, antiepileptic drugs.

O termo epilepsia se refere a um distúrbio da atividade cerebral caracterizada pela ocorrência periódica e espontânea de atividade elétrica altamente sincronizada, acompanhada de manifestações comportamentais ${ }^{1}$. Portanto, a epilepsia não é uma doença específica ou uma única síndrome, ela representa um grupo complexo de distúrbios decorrentes de funções cerebrais alteradas que podem ser secundárias a um grande número de processos patológicos². Acredita-se que dos novos casos de epilepsia a cada ano, $30-40 \%$ dos pacientes serão refratários às atuais terapias farmacológicas e conseqüentemente, apresentarão crises epilépticas recorrentes pelo resto de suas vidas ${ }^{3}$. As crises epilépticas são fenômenos clínicos transitórios, decorrentes da descarga excessiva e sincronizada da rede neuronal. Essas crises podem surgir espontaneamente ou ser desencadeadas por situações como: febre, distúrbio eletrolítico, intoxicação, doenças degenerativas e alterações vasculares ${ }^{4}$.

Estudos da prevalência e da incidência das epilepsias encontrados na literatura apresentam índices com grande variabilidade. Têm sido descritos valores de prevalência entre 0,9 e 57 casos/1000 habitan- tes e de incidência entre 26 e 190 casos/100000 habitantes. No entanto, essas variações podem ser resultantes de características metodológicas diversas, tais como: utilização de diferentes critérios diagnósticos, de classificação, de diferentes métodos de seleção de casos e definições das epilepsias ${ }^{1,5-7}$. No Brasil, um estudo recente demonstrou que a prevalência das epilepsias foi 186 por 1000 habitantes $^{8}$. A alta incidência das epilepsias nos países em desenvolvimento é decorrente da deficiente assistência pré-natal e maternal, alto índice de prematuridade, desnutrição, traumas durante o parto, convulsões febris da infância e de infecções, particularmente as decorrentes de parasitismo ${ }^{6,9}$. Além disso, a alta incidência e prevalência das epilepsias provocam repercussões nos aspectos sócio-econômicos ${ }^{10}$, na medida em que aumentam os custos econômicos diretos (provenientes dos gastos médicos, drogas e hospitalizações) e indiretos da doença (perda de produção econômica por desemprego, licença médica ou morte prematura) $)^{11}$. Agravando os problemas sócio-econômicos, o custo médio das drogas antiepilépticas é $25 \%$ mais alto que nos Estados Unidos e $145 \%$ mais alto que na Euro-

${ }^{1}$ Professor Adjunto da Disciplina de Neurologia Experimental da Universidade Federal de São Paulo/Escola Paulista de Medicina, São Paulo SP, Brasil (UNIFESP/EPM); ${ }^{2}$ Professora Adjunta de Neurologia da Universidade de Mogi das Cruzes SP, Brasil (UMC); ${ }^{3}$ Professor Adjunto do Departamento de Fisiologia da Universidade Federal de São Paulo/Escola Paulista de Medicina (UNIFESP/EPM); ${ }^{4}$ Professor Titular da Disciplina de Neurologia Experimental da Universidade Federal de São Paulo/Escola Paulista de Medicina (UNIFESP/EPM).

Recebido 13 Outubro 2006, recebido na forma final 12 Dezembro 2006. Aceito 6 Março 2007.

Dr. Fulvio Alexandre Scorza - Rua Botucatu 862 - Edifício Leal Prado / Disciplina de Neurologia Experimental - 04023-900 São Paulo SP - Brasil. E-mail: scorza.nexp@epm.br 
pa. Além disso, os novos fármacos antiepilépticos são $86 \%$ mais caros que nos Estados Unidos e $55 \%$ mais caros que na Europa ${ }^{12}$.

A primeira proposta para a classificação das crises epilépticas foi anunciada em 1969 por Henri Gastaut $^{13}$, baseada em critérios clínicos, eletrencefalográficos, substrato anatômico, etiologia e idade de ocorrência das crises. No entanto, com a introdução de novas técnicas, exigiu-se a revisão dessa classificação proposta em 1969 por Gastaut, culminando na Classificação Internacional das Crises Epilépticas ${ }^{14,15}$

Segundo a classificação das síndromes epilépticas da Liga Internacional Contra a Epilepsia (ILAE), as crises estão divididas em generalizadas e parciais, de acordo com a região cortical de origem e com a presença ou não de alteração de consciência durante o episódio. As crises generalizadas são aquelas que as descargas epilépticas envolvem ambos os hemisférios cerebrais simultaneamente, desde o início da crise, enquanto que nas crises parciais a atividade epiléptica está limitada a uma área focal do cérebro. As crises generalizadas são ainda divididas em tônico-clônica, de ausência, mioclônica, tônica, atônica e crises clônicas. As crises parciais são divididas em parciais simples e parciais complexas (de acordo com a preservação ou alteração da consciência). As crises parciais simples podem ser subdivididas em motoras, sensitivo ou sensoriais, vegetativas ou autonômicas e psíquicas. As crises parciais complexas podem não apresentar automatismos, ou cursar com automatismos simples, complexos ou posturas distônicas ${ }^{14,15}$.

Muitos avanços ocorreram na área da epileptologia, desde que as classificações de 1981 e 1989 foram adotadas, principalmente devido aos avanços das técnicas de imagem (ressonância magnética), vídeo-eletroencefalografia e a uma melhor compreensão das bases neuroquímicas e genéticas da epilepsia. Tais mudanças motivaram a revisão do sistema de classificação. De acordo com a proposição da ILAE de $2001^{16}$, mostra que a classificação é uma lista dos diferentes tipos de crises que são agora consideradas entidades diagnósticas. As crises epilépticas foram então dividas em três subgrupos: 1) crises isoladas ou autolimitadas (crises generalizadas ou crises focais), 2) crises contínuas, configurando o status epilepticus - SE (SE generalizado ou SE focal) e 3) crises reflexas, em que os fatores precipitantes podem desencadear crises focais ou generalizadas. Esta proposta ocorreu devido à necessidade de um sistema mais abrangente que viabilizasse a categorização das epilepsias sob vários aspectos ${ }^{17}$.

Em 2005, a ILAE propôs novas definições para os termos crise epiléptica e epilepsia, visando expressar o significado e as características essenciais desses dois termos. De acordo com a nova proposição, crise epiléptica é uma ocorrência transitória de sinais e/ou sintomas devido à atividade anormal excessiva ou atividade neuronal sincrônica no cérebro. Na mesma proposta, as epilepsias são definidas como um distúrbio do cérebro caracterizado pela predisposição em gerar crises epilépticas em condições neurobiológicas, psicológicas, cognitivas e conseqüências sociais dessa condição. Além disso, a caracterização de epilepsia requer a ocorrência de pelo menos uma crise epiléptica ${ }^{18}$. Também foi proposta nesta nova classificação a utilização preferencial do termo focal ao termo parcial ${ }^{18}$.

Dentre todos os tipos de epilepsias, os dados epidemiológicos mostram que a mais comum é a epilepsia do lobo temporal (ELT), que ocorre em cerca de $40 \%$ de todos os casos de epilepsias ${ }^{5}$ e é a forma mais comum nos adultos ${ }^{19}$.

A ELT é responsável por crises parciais complexas ${ }^{5}$, com correlação clínico-eletrencefalográfica bem definida, originadas nas estruturas límbicas temporais mesiais (epilepsia mesial temporal) ou na convexidade do lobo temporal (neocortical). O substrato patológico mais freqüentemente encontrado é a esclerose hipocampal que ocorre em $65 \%$ dos casos de ELT ${ }^{20}$. A esclerose hipocampal refere-se a uma perda acentuada de neurônios hilares e células piramidais no Corno de Ammon (CA), nas subregiões de CA1, CA3, e regiões do giro denteado do hipocampo ${ }^{21,22}$, com relativa preservação das células granulares e neurônios piramidais de CA2, ${ }^{23-25}$, acompanhada de glio$\mathrm{se}^{26}$ e atrofia da formação hipocampal. No entanto, outras estruturas também estão envolvidas, como a amígdala, o giro parahipocampal e o córtex entorrinal ${ }^{27}$. Além disso, diversas alterações estruturais também podem ser encontradas isoladamente ou em associação com a esclerose hipocampal como: tumores, displasias, calcificações, malformações vasculares, entre outras ${ }^{28,29}$.

\section{MORTE SÚBITA NAS EPILEPSIAS}

O fenômeno de morte súbita nas epilepsias (SUDEP) tem sido proposto desde $1910^{30}$. No entanto, para um melhor entendimento desse fenômeno, especialistas propuseram no congresso internacional sobre epilepsia e morte súbita, ocorrido em Londres em 1996, uma definição para tal evento: o óbito deve ocorrer de maneira não traumática, sem afogamento, pode ter ou não relatos de crise, excetuando-se status epilepticus, e os exames realizados após a morte não podem revelar causas anatômicas ou toxicológicas para a morte ${ }^{31}$. Apesar desta definição, muitas 
outras têm sido propostas e utilizadas ${ }^{32,33}$, no entanto, todas compartilham a problemática de que nem sempre é possível classificar cada caso individualmente, devido à falta de informações sobre as circunstâncias do óbito e pela dificuldade de realização de necropsias em diversos países.

A incidência da SUDEP tem sido estimada de $0,35 / 1000$ pessoas/ano em estudos populacionais ${ }^{33}$, 0,5-1.4/1000 pessoas/ano em indivíduos com epilepsia controlada ${ }^{34}, 3,5 / 1000$ pessoas/ano em ensaios clínicos para epilepsias focais ${ }^{35}, 5,9 / 1000$ pessoas/ano em centros de referência de epilepsia ${ }^{36}$ e 9,3/1000 pessoas/ano em candidatos para tratamento cirúrgico para as epilepsias ${ }^{37}$. A incidência da SUDEP difere em diversos estudos devido a diferenças das populações estudadas, níveis de documentação e da definição da SUDEP utilizada ${ }^{33,38,39}$.

Apesar desses dados epidemiológicos, alguns estudos têm identificado possíveis fatores de risco para a SUDEP, mas até o momento, nenhum mecanismo para este fenômeno foi claramente elucidado ${ }^{40}$. Apesar destas considerações, alguns fatores podem ser considerados de risco para a SUDEP: idade ${ }^{35}$; início precoce das epilepsias ${ }^{41}$; tempo de duração das epilepsias ${ }^{42}$; não controle e freqüência das crises epilépticas $^{42,43}$; tipos de crises epilépticas e regime de drogas antiepilépticas adotado ${ }^{44,45}$.

\section{ALTERAÇÕES CARDIOVASCULARES NAS EPILEPSIAS}

Como citado anteriormente, a ocorrência de morte súbita em pacientes com epilepsia não é tão rara quanto se acreditava. Apesar do mecanismo patofisiológico da SUDEP ser desconhecido, uma possível explicação é que este seja de origem cardiogênica. Dessa forma, estudos morfológicos e funcionais do coração têm sido realizados com o intuito de desvendar o fenômeno da SUDEP.

Do ponto de vista morfológico, alterações cardiovasculares têm sido demonstradas em pacientes com epilepsia que morreram subitamente. Os trabalhos nessa linha de investigação tiveram início na década de 70 e se estendem até os dias atuais. Quando necropsiado, o tecido cardíaco de pacientes com epilepsia apresenta alterações micro e macroscópicas que podem contribuir para a SUDEP, tais como: dilatação e hipertrofia cardíaca, fibrose nas paredes de pequenas artérias coronárias, atrofia dos cardiomiócitos, degeneração miofribilar, infiltração leucocitária que sugere miocardite focal e alterações morfológicas do sistema de condução cardíaco ${ }^{44,46-49}$. Frente a estes achados, especula-se que a hipoxemia repetida e/ou o aumento dos níveis de catecolaminas duran- te as crises epilépticas sejam fatores etiológicos responsáveis por tais alterações, uma vez que as mesmas também são verificadas em pacientes que evoluíram para óbito devido a cardiomiopatia isquêmica ${ }^{46,48,50}$. Além disso, algumas estratégias têm sido adotadas para detectar lesões do tecido cardíaco in vivo em pacientes com epilepsia. Para tal, algumas proteínas podem ser utilizadas como possíveis marcadores, como é o caso da troponina, cujo aumento plasmático de sua concentração pode ser considerado um importante indicador de lesão do tecido cardíaco ${ }^{51}$. Nesse sentido, Woodruff e colaboradores ${ }^{52}$ avaliando os níveis plasmáticos de troponina em 11 pacientes com epilepsia (antes e após o monitoramento das crises epilépticas), não encontraram alterações significativas nos níveis desta proteína, sugerindo dessa maneira, ausência de lesão miocárdica. No entanto, como as crises registradas nesse estudo foram de curta duração e capazes de induzir apenas uma taquicardia moderada, os autores acreditam que tais eventos não seriam suficientes para causar lesão miocárdica. Seguindo essa lógica, nosso grupo de pesquisa avaliou a concentração de troponina I em 40 pacientes com ELT refratária e que foram submetidos à ressecção cirúrgica. Nossos resultados indicam que mesmo na ausência do controle de crises após tratamento cirúrgico, os pacientes avaliados não apresentaram indícios de lesão do miocárdio, uma vez que os níveis plasmáticos de troponina I se encontraram dentro da faixa de normalidade. Apesar disso, o papel morfológico do coração na SUDEP não pode ser descartado, já que algumas lesões, embora não sendo capazes de alterar os níveis séricos de troponina I, podem ser suficientes na gênese de focos arritmogênicos ${ }^{53}$.

Do ponto de vista funcional, muitos trabalhos têm investigado possíveis alterações do sistema cardiovascular durante os períodos ictal e interictal de pacientes com epilepsia. Durante as crises epilépticas, a avaliação concomitante do EEG/ECG tem demonstrado que as crises epilépticas estão diretamente associadas com um aumento da freqüência cardíaca. Marshal e colaboradores $^{54}$ avaliaram 12 pacientes que foram monitorados através de video-EEG e ECG simultaneamente, observando a ocorrência de taquicardia durante as crises epilépticas. Paralelamente, Zijlmans e colaboradores ${ }^{55}$ observaram que ocorre um aumento na freqüência cardíaca de pelo menos 10 batimentos por minuto imediatamente antes do início das crises em $93 \%$ dos pacientes e em $23 \%$ das crises ( $49 \%$ dos pacientes), a taquicardia precedeu o início clínico e eletrográfico das crises. Tigaran e colaboradores ${ }^{56}$ demonstraram que $40 \%$ dos pacientes com epilepsia apresentaram depressão do segmento ST durante ou 
logo após a crise epiléptica e um outro estudo mostrou uma lentificação do prolongamento QT simultaneamente ao aparecimento de alterações eletrográficas detectadas no EEG, sendo que essas alterações cardíacas foram mais pronunciadas nos pacientes que morreram subitamente quando comparados com os que não evoluíram para óbito ${ }^{57}$. Por outro lado, é interessante frisarmos que alguns dados clínicos sugerem o envolvimento do sistema nervoso parassimpático durante as crises epilépticas, evidenciado por bradicardia sinusal ${ }^{58}$, a qual pode ser conseqüência da apnéia registrada durante as crises $^{59,60}$.

Além das alterações verificadas durante as crises epilépticas, alguns estudos têm relatado anormalidades funcionais do sistema cardiovascular em pacientes com epilepsia no período interictal. Nesse sentido, um estudo no final da década de 80 avaliou concomitantemente o EEG/ECG de 338 pacientes com epilepsia com o intuito de monitorar a presença de arritmias nos mesmos. Os autores verificaram que $5 \%$ dos pacientes apresentavam alterações do ritmo, embora essa taxa de anormalidade não difira daquelas encontradas em adultos saudáveis e assintomáticos ${ }^{61}$. Em 1993, Drake e colaboradores ${ }^{62}$ verificaram um aumento da freqüência cardíaca e da duração do prolongamento QT de 75 pacientes com epilepsia quando submetidos ao ECG, no entanto, estas alterações não se estavam fora dos padrões de normalidade. Além disso, os autores relataram que os pacientes com crises parciais complexas e crises secundariamente generalizadas apresentam uma maior freqüência ventricular e duração do intervalo QT quando comparados com indivíduos com epilepsia generalizada ${ }^{62}$. Recentemente, pesquisadores dinamarqueses utilizaram testes cardiológicos (ECG, monitorização por Holter, ecocardiografia, teste ergométrico e cintilografia miocárdica) com o intuito de avaliar possíveis alterações cardiovasculares em 23 pacientes com epilepsia refratária ${ }^{56}$. Nesse sentido, $40 \%$ dos pacientes avaliados apresentaram depressão do segmento ST, o qual foi associado com o aumento da freqüência cardíaca durante as crises epilépticas. Sendo assim, os autores concluíram que um processo de isquemia cardíaca pode ocorrer nos pacientes com epilepsia e que são refratários ao tratamento farmacológico ${ }^{56}$.

\section{MEDICAMENTOS ANTIEPILÉPTICOS E FUNÇÃO CARDIOVASCULAR}

A maioria dos estudos relaciona o fenômeno da SUDEP a eventos arritmogênicos. No entanto, diversos grupos de pesquisa têm demonstrado que os medicamentos antiepilépticos também são capazes de alterar a função cardiovascular ${ }^{63}$. Dentre todas as drogas utilizadas no tratamento das epilepsias e que possivelmente estão associadas com alterações do sistema cardiovascular, a carbamazepina ocupa um lugar de destaque. A carbamazepina aumenta o tônus simpático do sistema nervoso autônomo ${ }^{64}$, diminui a condução atrioventricular, podendo assim aumentar o risco de arritmias ${ }^{65}$, pode causar secreção inapropriada do hormônio antidiurético, desencadeando hiponatremia, fenômeno que pode estar associado com o quadro de morte súbita nas epilepsias ${ }^{66}$. Além disso, a carbamazepina foi implicada em uma série de 14 casos de SUDEP, em que $85 \%$ destes pacientes foram tratados com este fármaco ${ }^{67}$. Um trabalho recente demonstrou que a retirada abrupta de carbamazepina pode facilitar o aparecimento de SUDEP, por aumento da atividade simpática durante o sono, evidenciada por alterações nas taxas de variabilidade cardíaca ${ }^{68}$. Nessa mesma linha de raciocínio, outras drogas antiepilépticas também têm sido avaliadas. Na década de 80, demonstrou-se que a fenitoína apresenta propriedades antiarrítmicas ${ }^{63}$, no entanto, recentemente, alguns trabalhos foram realizados com o intuito de avaliar os efeitos da administração oral de fenitoína, fenobarbital e ácido valpróico sobre eventuais processos de arritmias e não foram observadas alterações no ritmo cardíaco ${ }^{69}$. Os benzodiazepínicos (ex. clonazepam) têm sido associados com o aumento do risco de morte súbita em crianças, principalmente com encefalopatias associadas $^{70}$. Esse fenômeno pode estar relacionado a quadros de disfagia, infecções recorrentes do trato respiratório (por hipersecreção brônquica), refluxo gastroesofágico e aspiração, especialmente pelo aumento da salivação, um efeito colateral importante desses medicamentos ${ }^{71}$. Vale a pena salientar que os estudos que relacionam drogas antiepilépticas e SUDEP apresentam limitações, uma vez que nesses trabalhos foram avaliados somente os efeitos proporcionados pelas drogas antiepilépticas per se, sem a associação com outras drogas que eventualmente podem estar sendo utilizadas pelos pacientes, principalmente nos que são refratários à terapia farmacológica e apresentam co-morbidades psiquiátricas.

\section{CONCLUSÕES}

Juntamente com o status epilepticus, a SUDEP é a principal causa de morte entre os pacientes com epilepsia, especialmente naqueles refratários ao tratamento farmacológico. Como já salientado, a SUDEP deve estar associada com alterações da função cardiovascular. Dessa maneira, seria pertinente que, após o diagnóstico do neurologista, os pacientes com epilepsia fossem avaliados por um médico cardiolo- 
gista, para que este profissional investigue a história clínica, o exame físico do sistema cardiorespiratório, a atividade elétrica e funcional do coração destes indivíduos. Sendo assim, acreditamos ser de fundamental importância à interação entre esses profissionais médicos (neurologistas e cardiologistas) no acompanhamento dos pacientes com epilepsia com maiores fatores de risco para a SUDEP, no intuito de um meIhor entendimento dos fenômenos cardiovasculares responsáveis pela morte súbita nas epilepsias, visando a sua prevenção.

\section{REFERÊNCIAS}

1. Mcnamara JO. Cellular and molecular basis of epilepsy. J Neuroscience 1994;14:3413-3425.

2. Guerreiro CAM, Guerreiro MM, Cendes F, Cendes IL (Eds). Considerações gerais em epilepsia. São Paulo: Lemos Editorial, 2000:1-10.

3. Kwan P, Sander JW. The natural history of epilepsy: an epidemiological view. J Neurol Neurosurg Psychiatry 2004;75:1376-1381.

4. Pedley TA, Bazil CW, Morrell MJ. Epilepsy. In Rowland LP (Ed). Merritt's neurology. Philadelphia: Lipincott Williams \& Wilkins, 2000: 663-641.

5. Hauser WA, Kurland LT. The epidemiology of epilepsy in Rochester, Minnesota, 1935 though 1937. Epilepsia 1975;16:1-66.

6. Sander JW, Shorvon SD. Incidence and prevalence studies in epilepsy and their methodological problems: a review. J Neurol Neurosurg Psychiatry 1987;50:829-839.

7. Jallon P, Goumaz M, Haengelli C, Morabia A. Incidence of first epileptic seizure in the canton of Geneva, Switzerland. Epilepsia 1997;38:547552.

8. Borges MA, Min LL, Guerreiro CA, et al. Urban prevalence of epilepsy: populational study in São José Rio Preto, a medium-sized city in Brazil. Arq Neuropsiquiatr 2004;62:199-204.

9. Fernandes MJ, Dube C, Boyet S, Marescaux C, Nehlig A. Correlation between hypermetabolism and neuronal damage during status epilepticus induced by lithium and pilocarpine in immature and adult rats. J Cereb Blood Flow Metab 1999;19:195-209.

10. Osuntokun BO, Adeuja AOG, Nottidge VA, Schoenberg, BS. Prevalence of the epilepsies in nigerian africans: a community-based study. Epilepsia 1987;28:272-279.

11. Robinson R. Cost benefit analisys. BMJ 1993;307:924-926.

12. Kochen S. Costing of antiepileptic drugs. Lancet 1996;347:266.

13. Gastaut H. Clinical and electroencephalographic classification of epileptic seizures. Epilepsia 1969;10:2-13.

14. ILAE - International League Against Epilepsy. Commission on Classification and Terminology. Proposal for revised clinical and electroencephalographic classification of epileptic seizures. Epilepsia 1981; 22:489-501.

15. ILAE - International League Against Epilepsy. Commission on Classification and Terminology. Proposal for revised classification of epilepsies and epileptic syndromes. Epilepsia 1989;30:389-399.

16. Engel J Jr. International League Against Epilepsy (ILAE). A proposed diagnostic scheme for people with epileptic seizures and with epilepsy: report of the ILAE Task Force on classification and terminology. Epilepsia 2001;42:796-803.

17. Engel J Jr. Mesial temporal lobe epilepsy: what have we learned? Neuroscientist 2001;7:340-352.

18. Fisher RS, van Emde Boas W, Blume W, et al. Epileptic seizures and epilepsy: definitions prpposed by the ILAE and the Internatinal Bureau for Epilepsy (IBE). Epilepsia 2005;46:470-472.

19. Walczak TS. Neocortical temporal lobe epilepsy: characterizing the syndrome. Epilepsia 1995;36:633-635.

20. Mathern GW, Babb TL, Armstrong DL. Hippocampal sclerosis. In Engel J Jr, Pedley TA (Eds). Epilepsy: a comprehensive textbook. Philadelphia: Lippincott-Raven, 1997:133-155.

21. Levesque MF, Nakasato N, Vinters HV, Babb TL. Surgical treatment of limbic epilepsy associated with extrahippocampal lesions: the problem of dual pathology. J Neurosurg 1991;75:364-370.

22. Meldrum BS. Pharmacological approaches to the treatments of epilepsy. In Meldrum BS, Porter RJ (Eds). New anticonvulsant drugs: currents problems in epilepsy. London: John Libbey, 1986;4:17-30.
23. Babb TL, Brown WJ. Pathological findings in epilepsy. In Engel J Jr (Ed). Surgical treatment of the epilepsies. New York: Raven Press, 1987:511540 .

24. Babb TL, Brown WJ, Pretorius JK, Davenport CJ, Lieb JP, Crandall PH. Temporal lobe volumetric cell densities in temporal lobe epilepsy. Epilepsia 1984;25:729-740.

25. Mouritzen-Dam A. Epilepsy and neuron loss in the hippocampus. Epilepsia 1980;21:617-629.

26. Wolf HK, Wiestler OD. Surgical pathology of chronic epileptic seizures disorders. Brain Pathol 1993;3:371-380.

27. Gloor P. Mesial temporal sclerosis: historical background and an overview from a modern perspective. In Luders H (Ed). Epilepsy surgery. New York: Raven Press, 1991:689-703.

28. Kuzniecky R, de la Sayette V, Ethier R, Melanson D, Andermann F, Berkovic S. Magnetic resonance imaging in temporal lobe epilepsy: pathological correlations. Ann Neurol 1987;22:341-347.

29. Ho SS, Kuzniecky RI, Gilliam F, Faught E, Morawetz R. Temporal lobe developmental malformations and epilepsy: dual patology and bilateral hippocampal abnormalities. Neurology 1998;50:748-754.

30. Munson JF. Death in epilepsy. Med Rec 1910;77:58-62.

31. Nashef L. Sudden unexpected death in epilepsy: terminology and definitions. Epilepsia 1997;38(Suppl):S6-S8.

32. Nashef L, Brown S. Epilepsy and sudden death. Lancet 1996;348:13241325.

33. Ficker DM, So El, Shen WK, et al. Population-based study of the incidence of sudden unexplained death in epilepsy. Neurology 1998; 51:1270-1274.

34. Tennis P, Cole TB, Annegers JF, Leestma JE, Mcnutt M, Rajput A. Cohort study of incidence of sudden unexplained death in persons with seizure disorder treated with antiepileptic drugs in Saskatchewan, Canada. Epilepsia 1995;36:29-36.

35. Leestma JE, Annegers JF, Brodie MJ, et al. Sudden unexplained death in epilepsy: observations from a large clinical development program. Epilepsia 1997;38:47-55.

36. Nashef L, Fish DR, Sander JW, Shorvon SD. Incidence of sudden unexpected death in an adult outpatient cohort with epilepsy at a tertiary referral centre. J Neurol Neurosurg Psychiatry 1995;58:462-464.

37. Dasheiff RM. Sudden unexpected death in epilepsy: a series from an epilepsy surgery program and speculation on the relationship to sudden cardiac death. J Clin Neurophysiol 1991;8:216-222.

38. Annegers JF, Coan SP, SUDEP: overview of definitions and review of incidence data. Seizure 1999; 8:347-352.

39. Langan $Y$, Nashef L, Sander JW. Certification of deaths attributable to epilepsy. J Neurol Neurosurg Psychiatry 2002;73:751-752.

40. Langan Y. Sudden unexpected death in epilepsy (SUDEP): risk factors and case control studies. Seizure 2000;9:179-183.

41. Nilsson L, Farahmand BY, Persson PG, Thiblin I, Tomson T. Risk factors for sudden unexpected death in epilepsy: a case-control study. Lancet 1999;353:888-893.

42. Walczak TS, Leppik IE, D'Amelio M, et al. Incidence and risk factors in sudden unexpected death in epilepsy: a prospective cohort study. Neurology 2001;56:519-525.

43. Sperling MR, Feldman H, Kinman J, Liporace JD, O'Connor MJ. Seizure control and mortality in epilepsy. Ann Neurol 1999;46:45-50.

44. Kloster R, Engelskjon T. Sudden unexpected death in epilepsy (SUDEP): a clinical perspective and a search for risk factors. J Neurol Neurosurg Psychiatry 1999;67:439-444.

45. Nilsson L, Bergman U, Diwan V, Farahmand BY, Persson PG, Tomson T. Antiepileptic drug therapy and its management in sudden unexpected death in epilepsy: a case-control study. Epilepsia 2001;42:667-673.

46. Falconer BE, Rajs J. Post-mortem findings of cardiac lesions in epileptics: a preliminary report. Forensic Sci 1976;8:63-71.

47. Antoniuk SA, Oliva LV, Bruck I, Malucelli M, Yabumoto S, Castellano JL. Sudden unexpected, unexplained death in epilepsy autopsied patients Arq Neuropsiquiatr 2001; 59:40-45.

48. Natelson BH, Suarez RV, Terrence CF, Turizo R. Patients with epilepsy who die suddenly have cardiac disease. Arch Neurol 1998;55:857-860.

49. Opeskin K, Thomas A, Berkpvic SF. Does cardiac condution pathology contribute to sudden unexpected death in epilepsy? Epilepsy Res 2000;40: 17-24.

50. Dasheiff RM, Dickinson RN. Sudden unexpected death of epileptic patient due to cardiac arrythmia after seizure. Arch Neurol 1986;43:194-196.

51. Gibbons RJ, Valeti US, Araoz PA, Jaffe AS. The quantification of infarct size. J Am Coll Cardiol 2004;44:1533-1542.

52. Woodruff BK, Briton JW, Tigaran S, et al. Cardiac troponin levels following monitored epileptic seizures. Neurology 2003;60:1690-1692. 
53. Colugnati DB, Cukiert A, Arida RM, et al. Cardiac troponin I levels in patients with refractory temporal lobe epilepsy after cortico-amygdalo-hippocampectomy. Braz J Epilep Clin Neurophysiol, 2007;13:7-11.

54. Marshall DW, Westmoreland BF, Sharbrough FW. Ictal tachycardia during temporal lobe seizures. Mayo Clin Proc 1983;58:443-446.

55. Zijlmans M, Flanagan D, Gotman J. Heart rate changes and ECG abnormalities durring epileptic seizures: prevalence and definition of an objective clinical sign. Epilepsia 2002; 43: 847-854.

56. Tigaran MD, Molgaard H, Mcclealland R, Dam M, Jaffe AS. Evidence of cardiac ischemia during seizures in drug refractory epilepsy patients. Neurology 2003;60:492- 495.

57. Tavernor SJ, Brown SW, Tavernor RM, Gifford C. Electrocardiograph QT lengthening associated with epileptiform EEG discharges: a role in sudden unexplained death in epilepsy? Seizure 1996;5:79-83.

58. Kiok MC, Terrence CF, Fromm GM, Lavine S. Sinus arrest in epilepsy. Neurology 1986;36:115-116.

59. Nashef L, Walker F, Allen P, Sander JW, Shorvon SD, Fish DR. Apnoea and bradycardia during epileptic seizures: relation to sudden death in epilepsy. J Neurol Neurosurg Psychiatry 1996;60:297-300.

60. So EL, Sam MC, Lagerlund TL. Postictal central apnea as a cause of SUDEP: evidence from near-SUDEP incident. Epilepsia 2000;41:1494-1497.

61. Keilson MJ, Hauser WA, Magrill JP, Goldman M. EcG abnormalities in patients with epilepsy. Neurology 1987;37:1624-1626.
62. Drake MF, Raider CR, Kay A. Electrocardiography in epilepsy patients without cardiac symptoms. Seizure 1993;2:63-65.

63. Lathers CM, Schraeder PL. Autonomic dysfunction in epilepsy: characterization of autonomic cardiac neural discharge associated with pentylenetetrazol-induced epileptogenic activity. Epilepsia 1982;23:633-647.

64. Devinksy OD, Perrini K, Theodore WH. Interictal autonomic nervous system functions in patients with epilepsy. Epilepsia 1994;35:199-204.

65. Herzberg L. Carbamazepine and bradycardia. Lancet 1978;1:1097-1098.

66. Kloster R, Borresen HC, Hoff-Olsen P. Sudden death in two patients with epilepsy and the SIADH. Seizure 1998;7:419-420.

67. Timmings PL. Sudden unexpected death in epilepsy: is carbamazepine implicated? Seizure 1998;7:289-291.

68. Hennessy MJ, Tighe MG, Binnie CD, Nashef L. Sudden withdrawal of carbamazepine increases cardiac sympathetic activity in sleep. Neurology 2001;57:1650-1654.

69. Walczak T. Do antiepileptic drugs play a role in sudden unexpected death in epilepsy? Drug Saf 2003;26:673-683.

70. Sankar R, Holmes GL. Mechanisms of action for the commonly used antiepileptic drugs: relevance to antiepileptic drug-associated neurobehavioral adverse effects, J. Child Neurology 2004;19(Suppl):S6-S14.

71. Rintahaka PJ, Nakagawa JA, Shewmon DA, Kyyronen P, Shields WD. Incidence of death in patients with intractable epilepsy during nitrazepam treatment. Epilepsia 1999;40:492-496. 